\title{
Axion EFTs and Massive Chiral Gauge Fields through the Anomalous Lens
}

\author{
Quentin Bonnefoy, ${ }^{a}$ Luca Di Luzio, ${ }^{a, b, c}$ Christophe Grojean, ${ }^{a, d}$ Ayan Paul ${ }^{a, d}$ and \\ Alejo N. Rossia ${ }^{a, d, e, *}$ \\ ${ }^{a} \mathrm{DESY}$, \\ Notkestraße 85, 22607 Hamburg, Germany \\ ${ }^{b}$ Dipartimento di Fisica e Astronomia 'G. Galilei', Università di Padova, \\ Via F. Marzolo, 8 - 35131 Padova, Italy \\ ${ }^{c}$ INFN Sezione di Padova, \\ Via F. Marzolo, 8 - 35131 Padova, Italy \\ ${ }^{d}$ Institut für Physik, Humboldt-Universität zu Berlin, \\ Newtonstraße 15, 12489 Berlin, Germany \\ ${ }^{e}$ Department of Physics and Astronomy, University of Manchester, Oxford Road, Manchester M13 9PL, \\ United Kingdom \\ E-mail: quentin.bonnefoy@desy.de, luca.diluzio@unipd.it, \\ christophe.grojean@desy.de, ayan.paul@desy.de, alejo.rossia@desy.de
}

We study axion effective field theories (EFTs), with a focus on axion couplings to massive chiral gauge fields. We investigate the EFT operators that couple an axion with two gauge bosons, and we show that, when massive chiral gauge fields are present, such interactions do not entirely originate from the usual anomalous EFT terms. The lack of relation between couplings and anomalies is caused by the presence of chiral fermions in the UV. For the case of an axion interacting with the Standard Model (SM) $W$ and $Z$ bosons, we show how anomaly-based sum rules in the IR one can detect whether the axion couplings are given or not by anomalies. Furthermore, the violation of those sum rules becomes a signal of heavy chiral matter. As an illustration, we study a UV-complete chiral extension of the SM, containing an axion arising from an extended Higgs sector and heavy fermionic matter that obtains most of its mass by coupling to the Higgs doublets. We assess the viability of such a SM extension through electroweak precision tests, bounds on Higgs rates and direct searches for heavy charged matter. We show that at energies below the mass of the new chiral fermions, the model matches onto an EFT where the electroweak gauge symmetry is non-linearly realised.

\footnotetext{
*** The European Physical Society Conference on High Energy Physics (EPS-HEP2021), *** *** 26-30 July $2021 * * *$

*** Online conference, jointly organized by Universität Hamburg and the research center DESY ***
}

\footnotetext{
${ }^{*}$ Speaker
} 


\section{Introduction}

Axion-like particles, which we will call simply axions from here on, are popular BSM particle candidates (see Ref. [1] for a recent review). Their parameter space has been probed with astrophysical, cosmological and collider data, and this exploration will intensify in the near future. Axion effective field theories (EFTs) is the preferred framework to study their phenomenology due to the usual mass gap between the axion and the rest of the UV sector.

The coupling of the axion to gauge fields is of great phenomenological relevance. The leading order interaction term and its relation with mixed anomalies is well-known for the case of massless gauge bosons [2]. However, recent efforts indicate the presence of additional operators that couple the axion to massive gauge bosons [3-5]. Furthermore, the mixed anomalies might not be enough to uniquely determine the coupling.

Here, and in more detail in Ref. [4], we re-examine the axion couplings to massive gauge fields, either abelian or not, in axion EFTs. We clarify when they are given by mixed anomalies and find connections with the presence of chiral fermions in the UV. Finally, we present a minimal phenomenologically-relevant extension of the SM that realizes the most general EFT of an axion and EW gauge bosons.

\section{Axion EFTs with massive gauge fields}

In this section, we will review the relation between axion couplings to gauge bosons and anomalies. First, we will focus on couplings to abelian gauge bosons. The axion coupling to abelian gauge bosons in an EFT is given at leading order by the following dimension-5 operator:

$$
\mathcal{L}_{\mathrm{EFT}} \supset-g^{2} \frac{C}{16 \pi^{2} f} a F_{\mu \nu} \tilde{F}^{\mu \nu}
$$

where $g$ is the gauge coupling constant of the gauge field $A$ with field strength $F_{\mu \nu}, \tilde{F}^{\mu \nu} \equiv \frac{\epsilon^{\mu \nu \rho \sigma}}{2} F_{\rho \sigma}$, $f$ is a dimensionful scale and $C$ a number. The axion $a$ is understood as the Nambu-Goldstone boson $(\mathrm{GB})$ of a spontaneously broken $\mathrm{PQ}$ symmetry $\mathrm{U}(1)_{\mathrm{PQ}}$, under which the gauge boson is uncharged. Similarly, the axion is neutral under the gauge symmetry $U(1)_{A}$.

This operator is generated by a loop of heavy fermions that are charged under both PQ and $U(1)_{A}$ symmetries and that have been integrated out of the EFT. We define the mixed anomaly polynomial $D^{\mathrm{PQ}, A A}=\sum_{\mathrm{LH} \psi} q_{\psi}^{P Q}\left(q_{\psi}^{A}\right)^{2}-\sum_{\mathrm{RH} \psi} q_{\psi}^{P Q}\left(q_{\psi}^{A}\right)^{2}$, where LH (RH) $\psi$ are all the left(right)-handed heavy fermions that enter in the loop, $q_{\psi}^{P Q}$ is their PQ charge and $q_{\psi}^{A}$ is their gauge charge. The usual statement is that $C$ is the anomaly coefficient $D^{\mathrm{PQ}, A A}$ corresponding to those fermions, i.e. $C=D^{\mathrm{PQ}, A A}$. Hence, the amplitude involving one axion and two gauge fields is determined by anomalies.

Such a statement is true when the gauge field is massless, but not entirely when $A$ has a non-zero mass. In the latter case, one additional gauge invariant operator becomes available [4]. Then, we like to think of the EFT of an axion and a massive abelian gauge field as

$$
\mathcal{L} \supset-g^{2} \frac{D^{\mathrm{PQAA}}}{16 \pi^{2} f} a F_{\mu \nu} \tilde{F}^{\mu \nu}-g \frac{C-D^{\mathrm{PQ} A A}}{8 \pi^{2} f} \partial_{\mu} a\left(\frac{\partial_{\nu} \theta_{A}}{m_{A}}-g A_{\nu}\right) \tilde{F}^{\mu \nu},
$$


where $\theta_{A}$ is the NGB that provides the longitudinal degree of freedom for the massive $A$ field and $m_{A}$ is the mass of the latter. This way of writing the EFT shows explicitly that for a massive gauge field, $C$ is not necessarily equal to $D^{\mathrm{PQ}, A A}$ but anomaly matching still holds, because only the first term shifts under the PQ symmetry. Additionally, it makes it clearer how to extend the analysis to the non-abelian case.

Although the non-vanishing mass of $A_{\mu}$ is a necessary condition for the mismatch $C \neq D^{\mathrm{PQ}, A A}$, it is not sufficient. Instead, what is sufficient is that the integrated-out fermions are chiral with respect to the gauge field $A$. More precisely, their mass eigenstates must be chiral ${ }^{1}$. Notice that the chiral couplings ensure that $A$ is massive.

At the level of scattering amplitudes, Eq. (1) and Eq. (2) are completely equivalent, there is no difference in the phenomenology predicted by anomalous and non-anomalous terms. The only way to see the mismatch between coupling and anomalies is to measure $C$ and compare it with $D^{\mathrm{PQ}, A A}$, which has to be computed from the UV model. This feature remains true even with several abelian gauge fields.

From an UV point of view, where one has control over the UV model, one can choose the PQ symmetry due to an irreducible ambiguity in the assignment of PQ charges to the fermions, associated to unbroken vector-like symmetries (see Ref. [3,6] for the case of lepton and baryon numbers). This freedom can be used to enforce $C=D^{\mathrm{PQ}, A A}$, although with some limitations (see [4] for details). This freedom does not exist from a purely IR point of view and therefore the second term in Eq. (2) should always be included in the EFT.

Now, we turn our attention towards non-abelian gauge bosons. We will focus on EW gauge bosons since the generalization of Eq. (2) to the non-abelian case can not be done in detail without specifying the symmetry breaking pattern. Let us start by generalizing the operator in Eq. (1):

$$
\mathcal{L} \supset-\frac{g^{2} C_{W W}}{16 \pi^{2}} \frac{a}{f} W^{a} \tilde{W}^{a}-\frac{g^{\prime 2} C_{B B}}{16 \pi^{2}} \frac{a}{f} B \tilde{B} .
$$

We only included two coefficients to respect the $\mathrm{SU}(2)_{L} \times \mathrm{U}(1)_{Y}$ gauge symmetry. This agrees with the form of the mixed PQ anomalies with the SU(2) factor of the SM gauge group.

In addition, there exist non-abelian analogues of the second term in Eq. (2). For the SM, there are 3 of those PQ- and gauge-invariant operators [5]:

$$
\partial_{\mu} a \operatorname{Tr}\left(T V_{v}\right) \tilde{B}^{\mu v}, \quad \partial_{\mu} a \operatorname{Tr}\left(V_{v} \tilde{W}^{\mu v}\right), \quad \partial_{\mu} a \operatorname{Tr}\left(T V_{v}\right) \operatorname{Tr}\left(T \tilde{W}^{\mu v}\right),
$$

where we use the operators $V_{\mu}=D_{\mu} U U^{\dagger}$ and $T=U \sigma_{3} U^{\dagger}$. These are defined from the Goldstone matrix $U=e^{i \frac{\pi^{a}}{v} \sigma^{a}}$ and its covariant derivative $D_{\mu} U=\partial_{\mu} U-i g W_{\mu} U+i g^{\prime} B_{\mu} U \frac{\sigma_{3}}{2}[5,7]$. The $\sigma^{a}$ are the Pauli matrices and $v \approx 246 \mathrm{GeV}$ is the electroweak VEV.

The terms in Eq. 4 will appear with a non-vanishing WC only when fermions that are chiral with respect to the $Z$ and $W$ bosons have been integrated out and their WC are not related to mixed PQ-gauge anomalies. This establishes a clear connection between chiral matter and nonanomalous axion couplings [4]. Furthermore, the integration of chiral fermions leads to an EFT with a non-linearly realised EWSB [9].

\footnotetext{
${ }^{1}$ This means that the LH and RH components of the mass eigenstate couple differently to the gauge bosons and is not equivalent to having chiral UV charges. From here on, we will mean chiral mass eigenstates by chiral unless stated otherwise. For details, see Section 2 of Ref. [4]
} 
If the operators in Eq. (3) were the whole story, they would induce correlations between the different EFT operators when written in terms of the vector massive eigenstates. In fact, using the latter, Eq. (3) becomes

$$
-\frac{16 \pi^{2}}{e^{2}} \mathcal{L} \supset C_{\gamma \gamma} \frac{a}{f} F \tilde{F}+2 \frac{C_{Z \gamma}}{c_{W} s_{W}} \frac{a}{f} F \tilde{Z}+\frac{C_{Z Z}}{c_{W}^{2} s_{W}^{2}} \frac{a}{f} Z \tilde{Z}+\frac{2 C_{W W}}{s_{W}^{2}} W^{+} \tilde{W}^{-},
$$

where the coefficients read $C_{\gamma \gamma}=C_{W W}+C_{B B}, C_{Z \gamma}=c_{W}^{2} C_{W W}-s_{W}^{2} C_{B B}, C_{Z Z}=c_{W}^{4} C_{W W}+$ $s_{W}^{4} C_{B B}$, and where $c_{W}=\cos \left(\theta_{W}\right), s_{W}=\sin \left(\theta_{W}\right)$, and $t_{W}=\tan \left(\theta_{W}\right)$ with $\theta_{W}$ the Weinberg angle, and we denoted the photon and $Z$-boson field strengths by $F$ and $Z$, respectively. The four coefficients in Eq. (5) are determined by $C_{B B}$ and $C_{W W}$ only. Thus, there must be correlations among the processes involving one axion and two electroweak gauge fields. This is different from the abelian case, that would allow independent coefficients for each gauge field pair. Such correlations can take the form of sum rules between EFT coefficients ${ }^{2}$, for instance:

$$
C_{\gamma \gamma}+s_{W}^{-2}\left(1-t_{W}^{2}\right) C_{Z \gamma}-\frac{1}{s_{W}^{2} c_{W}^{2}} C_{Z Z}=0, \quad C_{\gamma \gamma}+s_{W}^{-2} C_{Z \gamma}-\left(1+t_{W}^{-2}\right) C_{W W}=0
$$

However, the terms in Eq. (4) also generate amplitudes involving one axion and two gauge bosons in addition to that of Eq. (3). They increase the parameter space of axion EFTs and generically violate sum rules such as Eq. (6).

The axion EFT discussed so far could be straightforwardly used as a part of the most general SM-axion EFT. Nevertheless, it is imperative to reflect on which fermions can be integrated out. If we consider only the SM fermions, the most general SM-axion effective Lagrangian up to $d=5$ contains SM-axion interactions captured by

$$
\mathcal{L}_{a}^{d \leq 5}=\frac{1}{2}\left(\partial_{\mu} a\right)^{2}-\frac{1}{2} m_{a}^{2} a^{2}+\frac{\partial^{\mu} a}{f} \sum_{\psi} \bar{\psi} \mathbf{C}^{(\psi)} \gamma_{\mu} \psi-\sum_{X=G, W, B} \frac{g_{s}^{2} C_{X X}}{16 \pi^{2}} \frac{a}{f} X \tilde{X},
$$

where the sum over $\psi$ is taken over the chiral fermions of the SM and $\mathbf{C}^{(\psi)}$ is a hermitian matrix in generation space. Here, the $C_{X X}$ coefficients are the anomaly coefficients of the PQ symmetry with the gauge group of gauge field $X$, when it is restricted to the SM fields only.

Nonetheless, in the SM, not even the top quark can be integrated out safely when considering the axion couplings to $W / Z$ bosons. Hence, if the axion couples to SM fields, one cannot rely on an EFT analysis for the axion couplings to massive gauge bosons. On the other hand, an interesting EFT limit exists if the axion couplings to $W / Z$ bosons are generated by extra fermions that are chiral under $S U(2)_{L} \times U(1)_{Y}$. The mass of those fermions is forced by experimental results to be at least several hundreds of $\mathrm{GeV}$, which allows us to integrate them out when discussing the axion couplings to $W / Z$ bosons.

In order to form the full SM-axion EFT below the mass scale of the aforementioned extra chiral fermions, one should add to the couplings in Eq. (7) the contributions of the heavy fermions which are integrated out. This brings additional terms, in particular couplings between the axion and the electroweak gauge fields which do not simply add up to the $C_{X X}$. During the rest of this work, we focus on the contribution of the heavy chiral fields.

\footnotetext{
${ }^{2}$ The sum-rules are understood to hold at energy scales where the $W$ and $Z$ bosons are dynamical degrees of freedom.
} 


\section{A minimal model with axions and chiral matter}

Following Ref. $[8,12,13]$, we build a minimal model that adds an axion and chiral fermions to the SM according to the following conditions: (i) no massless fermion after EW symmetry breaking, apart from SM gauge singlets; (ii) no gauge and Witten [10] anomalies; (iii) compatibility with Higgs coupling modifications; and (iv) no allowed bare mass terms. The bare mass terms could be either accidentally suppressed compared to the EW scale or forbidden by extra symmetries, for instance discrete gauge ones.

It can be seen that the minimal set of chiral fermions that fulfils those criteria is $[4,8]$

$$
\begin{array}{lll}
L_{1} \sim(1,2,+Y), & E_{1} \sim\left(1,1,+Y-\frac{1}{2}\right), & N_{1} \sim\left(1,1,+Y+\frac{1}{2}\right), \\
L_{2} \sim(1,2,-Y), & E_{2} \sim\left(1,1,-Y+\frac{1}{2}\right), & N_{2} \sim\left(1,1,-Y-\frac{1}{2}\right) .
\end{array}
$$

In this minimal setup, gauge anomaly cancellation is immediate since its field content is vector-like with respect to the SM gauge group. Nonetheless, we still consider it as chiral because we do not include bare mass terms and only use EWSB to generate their masses in such a way that the LH and RH components of the mass eigenstates couple differently.

The scalar sector of this model contains a complex SM singlet $\Phi$ and two Higgs doublets $H_{1,2} \sim\left(1,2, \frac{1}{2}\right)$. Their potential is $V\left(H_{1}, H_{2}, \Phi\right)=V_{\text {r.i. }}\left(\left|H_{1}\right|,\left|H_{2}\right|,|\Phi|,\left|H_{1}^{\dagger} H_{2}\right|\right)+\lambda H_{1}^{\dagger} H_{2} \Phi^{2}+$ h.c. , which includes all the re-phasing invariant terms allowed by gauge invariance plus a non-hermitian operator. The latter breaks the three re-phasing symmetries $\mathrm{U}(1)_{\Phi} \times \mathrm{U}(1)_{H_{1}} \times \mathrm{U}(1)_{H_{2}}$ into two linearly independent $\mathrm{U}(1)$ 's that we identify with $\mathrm{U}(1)_{Y} \times \mathrm{U}(1)_{P Q}$. The PQ charges of $H_{1}$ and $H_{2}$, $X_{1}$ and $X_{2}$ respectively, are constrained to fulfil $X_{1}-X_{2}=2$, where we have normalized the PQ charge of $\Phi$ as $X_{\Phi}=1$.

If only one of the Higgs doublets interacts with the 2 families of new chiral leptons, there would be no dimension-5 axion-gauge boson couplings in the EFT below the mass of the exotic fermions [4]. Hence, the Yukawa Lagrangian must include explicitly both doublets. We use the following Lagrangian:

$$
-\mathcal{L}_{Y}=y_{E_{1}} \bar{L}_{1} E_{1} H_{1}+y_{E_{2}} \bar{L}_{2} E_{2} \tilde{H}_{2}+y_{N_{1}} \bar{L}_{1} N_{1} \tilde{H}_{2}+y_{N_{2}} \bar{L}_{2} N_{2} H_{1}+\text { h.c. . }
$$

A different assignment of $H_{1,2}$ in Eq. (10) would amount to a different choice of PQ symmetry and modify the axion and/or Higgs boson phenomenology. We do not specify the Higgs fields assignment in the SM Yukawa couplings, we simply assume that it only involves $H_{1,2}$, and that it does not generate tree-level flavour-changing neutral currents. One possibility is to couple them as in the original DFSZ model [12,13], in which case Eq. (7) is simply the usual DFSZ axion-SM EFT.

The axion can be identified as a linear combination of the phases of the different scalars via usual methods (see Ref. [4] for details). Its couplings to the new chiral leptons are given by:

$$
-\mathcal{L}_{Y} \supset\left(e^{i X_{1} \frac{a}{f}}\right) \sum_{\psi=E_{1}, N_{2}} m_{\psi} \bar{\psi}_{L} \psi+\left(e^{-i X_{2} \frac{a}{f}}\right) \sum_{\psi=E_{2}, N_{1}} m_{\psi} \bar{\psi}_{L} \psi
$$

where we have decomposed the doublets as $L_{i}=\left(N_{i L}, E_{i L}\right)^{T}$, and defined the Dirac masses $m_{E_{i}}=y_{E_{i}} \frac{v_{1}}{\sqrt{2}}$ and $m_{N_{i}}=y_{N_{i}} \frac{v_{2}}{\sqrt{2}}$, where $i=1,2$. We could remove the axion field from the mass 
terms by redefining the fermion fields via a field-dependent chiral transformation. Nevertheless, it is easier to obtain the axion-gauge bosons couplings in the basis of Eq. (11).

Now, we derive the axion EFT below the mass of the new chiral leptons in the model presented before. First, we define the massive eigenstates $N_{i}=N_{i}+N_{L_{i}}, E_{i}=E_{i}+E_{L_{i}}$ and assume equal masses within a SU(2) doublet, i.e. $m_{N_{i}}=m_{E_{i}}$. Using the formulae in Ref. [4], the axion couplings in the EFT read

$$
\mathcal{L} \supset-g^{\prime 2} \frac{\left(1+12 Y^{2}\right)\left(X_{1}-X_{2}\right)}{96 \pi^{2}} \frac{a}{f} B \tilde{B}-g^{2} \frac{X_{1}-X_{2}}{96 \pi^{2}} \frac{a}{f} W^{a} \tilde{W}^{a}-g g^{\prime} \frac{X_{1}-X_{2}}{96 \pi^{2}} \frac{a}{f} B \tilde{W}^{3},
$$

where we used the same letter to refer to the gauge fields and to their field strengths.

The last term in Eq. (12) can not be reproduced by an UV PQ anomaly because there is no non-vanishing $\mathrm{U}(1)_{P Q} \times \mathrm{U}(1)_{Y} \times \mathrm{SU}(2)_{L}$ anomaly coefficient. More precisely, it does not match the usual ansatz in Eq. (3), but it can be obtained from a combination of the EFT terms in Eq. (4). The fact that these couplings do not derive solely from UV anomalies becomes evident when one finds out that the sum rules in Eq. (6) are violated as follows:

$$
C_{\gamma \gamma}+s_{W}^{-2}\left(1-t_{W}^{2}\right) C_{Z \gamma}-\frac{C_{Z Z}}{s_{W}^{2} c_{W}^{2}}=\frac{X_{1}-X_{2}}{12 c_{W}^{2} s_{W}^{2}}, \quad C_{\gamma \gamma}+s_{W}^{-2} C_{Z \gamma}-\left(1+t_{W}^{-2}\right) C_{W W}=\frac{X_{2}-X_{1}}{12 s_{W}^{2}} .
$$

The violation of these sum rules can be directly tested given the observation of the axion-gauge boson couplings and constitutes a smoking gun of the presence of a chiral heavy sector charged under the PQ symmetry. Therefore, the model considered here shows how the terms in Eq. (4) appear at dimension 5 in the Lagrangian when chiral fermions are integrated out, and are not suppressed by the mass of the heavy fermions. If we had allowed for bare mass terms for the fermions, these non-decoupling effects would have been absent.

If $m_{a}>2 m_{Z}$, all the decays of an axion to gauge bosons are allowed and one can rewrite the sum rules in terms of partial decay rates as follows:

$$
\text { SR-1: }\left[\frac{\Gamma(a \rightarrow Z Z)}{\Gamma(a \rightarrow \gamma \gamma)}-1-\frac{\left(t_{W}^{2}-1\right)^{2}}{2 t_{W}^{2}} \frac{\Gamma(a \rightarrow Z \gamma)}{\Gamma(a \rightarrow \gamma \gamma)}\right]^{2}-\frac{2\left(t_{W}^{2}-1\right)^{2}}{t_{W}^{2}} \frac{\Gamma(a \rightarrow Z \gamma)}{\Gamma(a \rightarrow \gamma \gamma)}=0,
$$

which is a relation between two quantities that can be traced on a plane. Another sum rule that follows from both identities in Eq. (6) is

$$
\text { SR-2: } \Gamma(a \rightarrow \gamma \gamma)+\frac{1}{2}\left(t_{W}^{-2}-1\right) \Gamma(a \rightarrow W W)-t_{W}^{-2} \Gamma(a \rightarrow Z Z)+\frac{1}{2}\left(1-t_{W}^{-2}\right) \Gamma(a \rightarrow Z \gamma)=0 .
$$

In both cases, we neglect the SM contribution to the partial decay rates. For the relation between the partial decay rates and the Wilson Coefficients, see Ref. [4]. These sum rules can be tested with low-energy measurements. If at least one of them is violated, we can conclude that the fermionic UV completion is chiral. The violation of the sum rules in our specific model is displayed in Fig. 1, where one can see that the model only satisfies both sum rules when $|Y| \rightarrow \infty$. We stress the caveat that SM loop contributions also violate the naive sum rules, so they should be taken into account.

Finally, we comment about other experimental constraints. The bounds from EWPO can be evaded easily in the custodial limit of the heavy chiral leptons. On the other hand, Higgs precision data imposes stringent constraints, since the new fermions modify the Higgs decay to photons and 


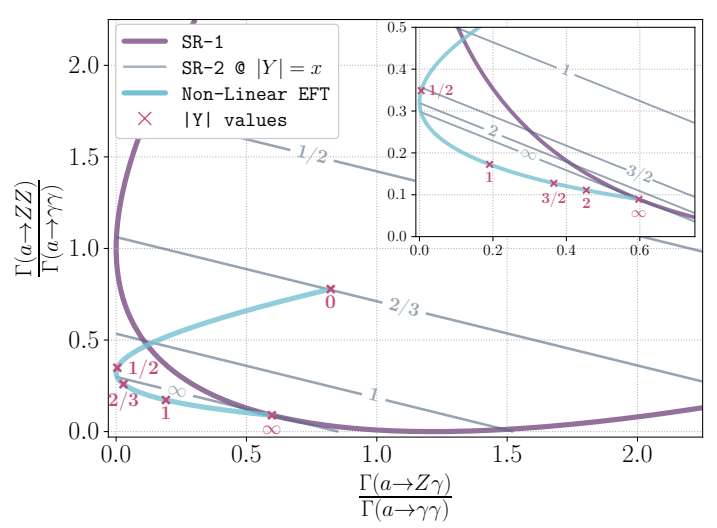

Figure 1: Plot of the ratio of decay rates $\frac{\Gamma(a \rightarrow Z Z)}{\Gamma(a \rightarrow \gamma \gamma)}$ as a function of the ratio of decay rates $\frac{\Gamma(a \rightarrow Z \gamma)}{\Gamma(a \rightarrow \gamma \gamma)}$, assuming that the decays are kinematically allowed. The sum rule SR-1 (SR-2) in Eq. (14) (Eq. (15)) corresponds to the purple (grey) curve. Each grey line then corresponds to different values of $Y$. Finally, the blue curve uses the couplings derived from our model.

to $Z \gamma$. In the alignment limit, the current measurements of $R_{\gamma \gamma}=\frac{\Gamma(h \rightarrow \gamma \gamma)}{\Gamma(h \rightarrow \gamma \gamma)_{S M}}$ allows only the region $1.43 \lesssim|Y| \lesssim 1.53$, which generates a deviation in $R_{Z \gamma}=\frac{\Gamma(h \rightarrow Z \gamma)}{\Gamma(h \rightarrow Z \gamma)_{S M}}$ big enough to be discovered or excluded at HL-LHC. Those constraints can be evaded in the so-called wrong-sign limit, which instead is affected by stringent constraints on the rate between the 2 Higgs VEVs [4]. The heavy chiral leptons can be produced at colliders via Drell-Yan and recent searches impose a lower bound on their mass of $M \gtrsim 720 \mathrm{GeV}$ for $Y=3 / 2$ in the custodial limit and depending on how degenerate the masses inside each doublet are $[4,11])$.

\section{Conclusions}

We investigated the axion couplings to massive chiral gauge fields in axion EFTs and their connections with UV models. Differently from the case of massless gauge fields, this coupling is not entirely captured by mixed anomalies. We provided a new understanding of this phenomenon, stressing that the key point is that the longitudinal part of massive gauge fields allows to write additional non-anomalous operators linking axions with the gauge field. The UV origin of those additional operators is rooted in the presence of chiral fermions.

In the case of non-abelian gauge bosons, the phenomenology is changed by the presence of the non-anomalous operators. This is clearly exemplified by the violation of the sum rules. Finally, we built a minimal extension of the SM with chiral fermions in which the most general axion EFT with massive chiral gauge fields is realized and showed explicitly how the non-anomalous operators are generated by chiral heavy fermions. 


\section{Acknowledgements}

We thank Emilian Dudas, Tony Gherghetta, Stefan Pokorski, Jérémie Quevillon and Pablo Quílez for useful discussions. This work is supported by the Deutsche Forschungsgemeinschaft under Germany's Excellence Strategy EXC 2121 "Quantum Universe" - 390833306. The work of C.G. and A.R. was also supported by the International Helmholtz-Weizmann Research School for Multimessenger Astronomy, largely funded through the Initiative and Networking Fund of the Helmholtz Association. The work of L.D.L. is supported by the Marie Skłodowska-Curie Individual Fellowship grant AXIONRUSH (GA 840791).

\section{References}

[1] L. Di Luzio, M. Giannotti, E. Nardi and L. Visinelli, "The landscape of QCD axion models," Phys. Rept. 870 (2020), 1-117 doi:10.1016/j.physrep.2020.06.002 [arXiv:2003.01100 [hep$\mathrm{ph}]]$.

[2] J. Steinberger, "On the Use of subtraction fields and the lifetimes of some types of meson decay,” Phys. Rev. 76 (1949), 1180-1186 doi:10.1103/PhysRev.76.1180

[3] J. Quevillon and C. Smith, Eur. Phys. J. C 79 (2019) no.10, 822 doi:10.1140/epjc/s10052-0197304-4 [arXiv:1903.12559 [hep-ph]].

[4] Q. Bonnefoy, L. Di Luzio, C. Grojean, A. Paul and A. N. Rossia, JHEP 07 (2021), 189 doi:10.1007/JHEP07(2021)189 [arXiv:2011.10025 [hep-ph]].

[5] I. Brivio, M. B. Gavela, L. Merlo, K. Mimasu, J. M. No, R. del Rey and V. Sanz, Eur. Phys. J. C 77 (2017) no.8, 572 doi:10.1140/epjc/s10052-017-5111-3 [arXiv:1701.05379 [hep-ph]].

[6] J. Quevillon and C. Smith, Phys. Rev. D 102 (2020) no.7, 075031 doi:10.1103/PhysRevD.102.075031 [arXiv:2006.06778 [hep-ph]].

[7] F. Feruglio, Int. J. Mod. Phys. A 8 (1993), 4937-4972 doi:10.1142/S0217751X93001946 [arXiv:hep-ph/9301281 [hep-ph]].

[8] N. Bizot and M. Frigerio, JHEP 01 (2016), 036 doi:10.1007/JHEP01(2016)036 [arXiv:1508.01645 [hep-ph]].

[9] T. Cohen, N. Craig, X. Lu and D. Sutherland, JHEP $\mathbf{0 3}$ (2021), 237 doi:10.1007/JHEP03(2021)237 [arXiv:2008.08597 [hep-ph]].

[10] E. Witten, Phys. Lett. B 117 (1982), 324-328 doi:10.1016/0370-2693(82)90728-6

[11] V. Khachatryan et al. [CMS], Phys. Rev. D 94 (2016) no.11, 112004 doi:10.1103/PhysRevD.94.112004 [arXiv:1609.08382 [hep-ex]].

[12] M. Dine, W. Fischler and M. Srednicki, Phys. Lett. B 104 (1981), 199-202 doi:10.1016/03702693(81)90590-6

[13] A. R. Zhitnitsky, Sov. J. Nucl. Phys. 31 (1980), 260 